\title{
Tuning of giant magnetoimpedance effect of amorphous and nanocrystalline microwires
}

\author{
Valentina Zhukova ${ }^{1,2}$, Ahmed Talaat ${ }^{1,2}$, Mihail Ipatov ${ }^{1,2}$, Alexandr Granovsky ${ }^{3}$ and Arcady \\ Zhukov ${ }^{1,2,4 *}$
}

\author{
${ }^{1}$ Dpto. de Fís. Mater., UPV/EHU San Sebastián 20009, Spain \\ ${ }^{2}$ Dpto. de Física Aplicada, EUPDS, UPV/EHU, 20018, San Sebastian, Spain \\ ${ }^{3}$ Faculty of Physics, Lomonosov Moscow State University, 11991 Moscow, Russian Federation \\ ${ }^{4}$ IKERBASQUE, Basque Foundation for Science, 48011 Bilbao, Spain \\ *corresponding author, E-mail: arkadi.joukov@ehu.es
}

\begin{abstract}
Composite structural materials containing periodic or random arrays of metallic wires often referred to as wire metamaterials have a strong dispersion of the dielectric function in the $\mathrm{GHz}$ frequency band. These metamaterials utilising thin ferromagnetic wires exhibiting magnetoimpedance effect present tuneability of the effective permittivity, by a weak magnetic field or a mechanical stress. We present our studies of the factors affecting soft magnetic properties and giant magnetoimpedance effect (GMI) in thin amorphous and nanocrystalline microwires. We showed that the magnetoelastic anisotropy is one of the most important parameters that determine magnetic softness and GMI effect of glass-coated microwires and annealing can be very effective for manipulation the magnetic properties of amorphous ferromagnetic glass-coated microwires. Considerable magnetic softening and increasing of the GMI effect is observed in Fe-rich nanocrystalline FINEMET-type glass-coated microwires after the nanocrystallization.
\end{abstract}

\section{Introduction}

Studies of innovative design of electromagnetic materials continue to be an important issue in microwave technology. A recent trend is to achieve adjustability in these structures. One of the perspective materials is the tuneable metamaterial utilising thin ferromagnetic wires exhibiting magnetoimpedance effect (MI) [1,2]. Our and some other groups work demonstrates that this leads to powerful adjustment of their electromagnetic properties, in particular, effective permittivity, by a weak magnetic field or a mechanical stress[1-3]. Composite structural materials containing periodic or random arrays of metallic wires often referred to as wire metamaterials have a strong dispersion of the dielectric function in the $\mathrm{GHz}$ frequency band. This behaviour could be of plasmonic or resonance type for long or short-cut wires, respectively $[1,2]$. In both cases, the real part of the permittivity can be negative in the dispersion region resulting in many fascinating effects including negative refraction and frequency selective band-gap or band-pass regimes [1-3].

In thin conducting wires the currents that are responsible for effective permittivity are constrained with the associated resonances determined by the geometrical parameters. The current resonances are damped due to the wire impedance which may increase greatly when the wire magnetisation is changed. This is known as giant magnetoimpedance (GMI) effect [4-6]. In soft magnetic amorphous wires subjected to an external magnetic field GMI is in the range of $100 \%$ even at frequencies of few GHz [4-6]. Increase in magnetic losses results in increase in the relaxation parameter which determines the frequency dispersion of the effective permittivity. In the case of plasmonic wire arrays, this will result in considerable decrease in the absolute value of the permittivity and will enhance the wave propagation. Similarly, in cut-wire composites, the increase in relaxation broadens the permittivity dispersion which may even show transformation from resonance to relaxation behaviour [1-3]. Therefore, in composites containing ferromagnetic wires exhibiting GMI effect at $\mathrm{GHz}$ frequencies the effective permittivity will depend on the wire magnetic properties via the corresponding dependence of its impedance.

Consequently tailoring of the GMI effect in magnetic wires is essential for performance of the tuneable metamaterial utilising thin ferromagnetic wires.

Recent few years studies of glass-coated magnetic microwires exhibiting excellent sift magnetic properties and Giant magnetoimpedance effect gained considerable attention[5-7]. These properties are related to cylindrical symmetry as well as to crystalline structure of magnetic wires.

On the other hand GMI effect and its magnetic field dependence are closely related to the internal and applied stresses [8-10]. Therefore both GMI and magnetic softness can be tailored by magnetic wires processing.

Consequently we present our recent studies of the factors affecting soft magnetic properties and giant magnetoimpedance (GMI) effect in thin amorphous wires. 


\section{Experimental details}

For the preparation of Co- and Fe-rich Finemet-type glasscoated microwires we employed modified Taylor-Ulitovsky technique and/or quenching-and-drawing method described elsewhere [5,6]. This method essentially consists of a simultaneous drawing of the composite microwire (metallic nucleus inside the glass capillary) through the quenching liquid (water or oil) jet onto rotating bobbins. More details on microwires preparation can be found elsewhere [5-8].

We studied glass-coated $\mathrm{Co}_{50.69} \mathrm{Fe}_{8.13} \mathrm{Ni}_{17.55} \mathrm{~B}_{13.29} \mathrm{Si}_{10.34}$ microwires $(d=12.8 \mu \mathrm{m}, \quad D=15.8 \quad \mu \mathrm{m}, \quad \rho=0.81) \quad$ and $\mathrm{Fe}_{70.8} \mathrm{Cu}_{1} \mathrm{Nb}_{3.1} \mathrm{Si}_{14.5} \mathrm{~B}_{10.6}(d=10.7 \mu \mathrm{m}, D=16.4 \mu \mathrm{m}, \rho=0.6)$. We have measured the magnetic field dependence of impedance, $\mathrm{Z}$, and GMI ratio, $\Delta \mathrm{Z} / \mathrm{Z}$, for as-prepared samples and after heat treatments $[5,6]$.

We used a specially designed micro-strip sample holder described elsewhere [5]. The sample holder was placed inside a sufficiently long solenoid that creates a homogeneous magnetic field, $H$. The sample impedance, $Z$, was measured using a vector network analyzer from reflection coefficient $S_{11}$. defined as:

The magneto impedance ratio, $\Delta Z / Z$, has been

$$
\Delta Z / Z=\left[Z(H)-Z\left(H_{\max }\right)\right] \cdot 100 / Z\left(H_{\max }\right),
$$

An axial $D C$-field with a maximum value $H_{\max }$ up to 8 $\mathrm{kA} / \mathrm{m}$ was supplied by magnetizing coils.

The frequency range for the diagonal impedance component has been measured from $1 \mathrm{MHz}$ up to $7 \mathrm{GHz}$.

Hysteresis loops have been determined by the flux-metric method described elsewhere $[5,8]$.

Crystalline structure and phase composition have been checked using a BRUKER (D8 Advance) X-ray diffractometer with $\mathrm{CuK \alpha}(\lambda=1.54 \AA)$ radiation.

The magnetostriction coefficient has been measured using the small angle magnetization rotation method (SAMR) method described elsewhere [11] and recently modified for the case of thin magnetic wires [12].

\section{Experimental results and discussion}

\subsection{Tailoring of magnetic properties and GMI effect in Co-rich amorphous microwires}

As can be seen from Fig. 1 typically as-prepared Corich microwires presents typical for Co-rich microwires quasi-linear hysteresis loops and XRD typical for amorphous structure (consisted of a diffuse halo without observation of any crystalline peak). A coercive field of both samples is about $4 \mathrm{~A} / \mathrm{m}$ that is typical for amorphous Co-based glass-coated microwires with low negative magnetostriction constant. The origin of such magnetic softness is related to the internal stresses arising during preparation of glass-coated microwires $[8,14]$. These stresses give rise to an easy magnetization direction
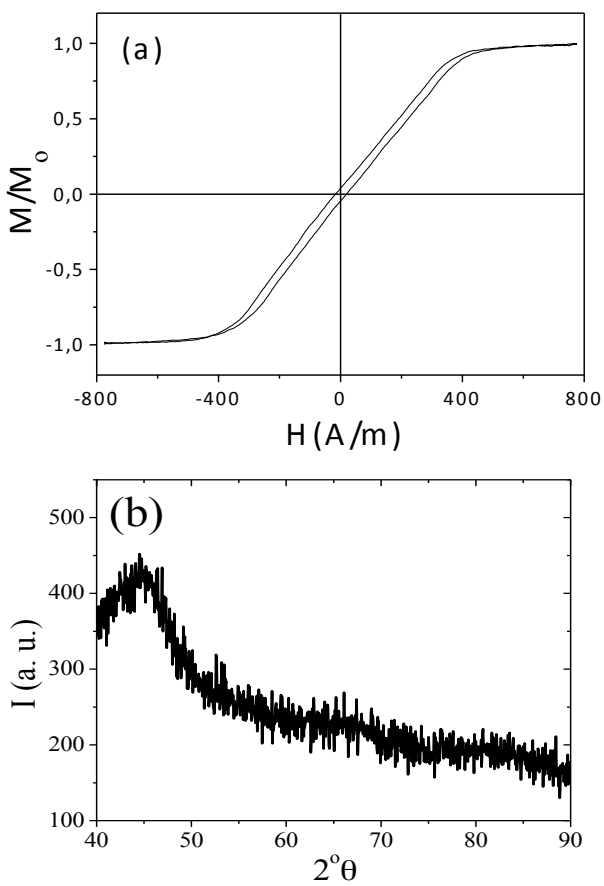

Figure 1: Hysteresis loop (a) and XRD patterns (b) of as-prepared $\mathrm{Co}_{50.69} \mathrm{Fe}_{8.13} \mathrm{Ni}_{17.55} \mathrm{~B}_{13.29} \mathrm{Si}_{10.34}$ microwire

perpendicular to the wire axis, leading to an alignment of the magnetic moments in the direction which is perpendicular (circumferential) to the wire axis.

The annealing even for quite short time and at low temperature leads to significant changing of the magnetic properties of both studied Co-rich microwires (Figs 2a-c).
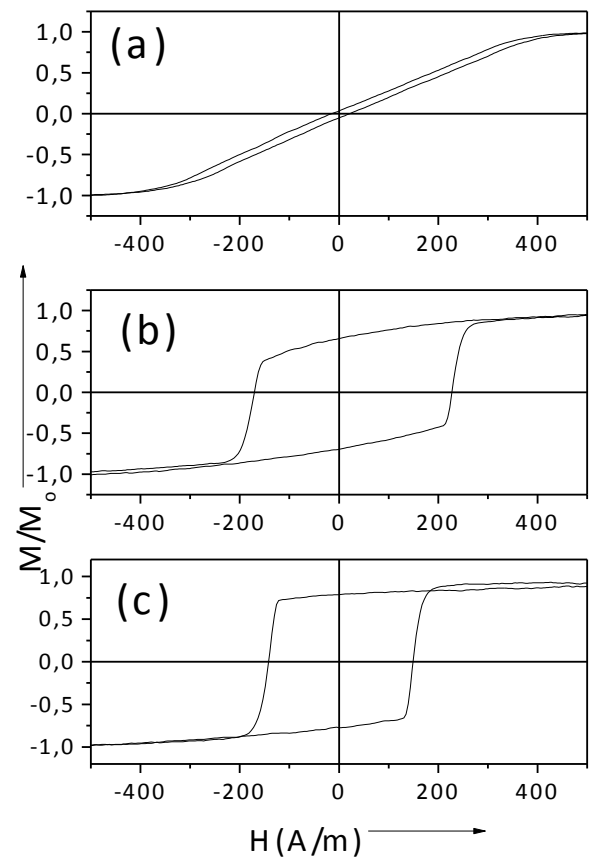

Figure 2: Hysteresis loops of as-prepared (a) and annealed for $5 \mathrm{~min}$ at $200^{\circ} \mathrm{C}$ (b) and $250^{\circ} \mathrm{C}$ (c) $\mathrm{Co}_{50.69} \mathrm{Fe}_{8.13} \mathrm{Ni}_{17.55} \mathrm{~B}_{13.29} \mathrm{Si}_{10.34}$ microwires 
Increasing of the annealing temperature the hysteresis loop becomes more rectangular: remanent magnetization rises with increasing of $T_{a n n}$, although coercivity, $H_{c}$, remains almost the same for all annealing conditions.

In spite of considerable magnetic hardening (a remarkable increasing of coercivity from 4 to $200 \mathrm{~A} / \mathrm{m}$ for $\mathrm{Co}_{50.69} \mathrm{Fe}_{8.13} \mathrm{Ni}_{17.55} \mathrm{~B}_{13.29} \mathrm{Si}_{10.34}$ sample), both as-prepared and annealed microwires at different annealing conditions present considerable GMI effect as shown in Fig 3.
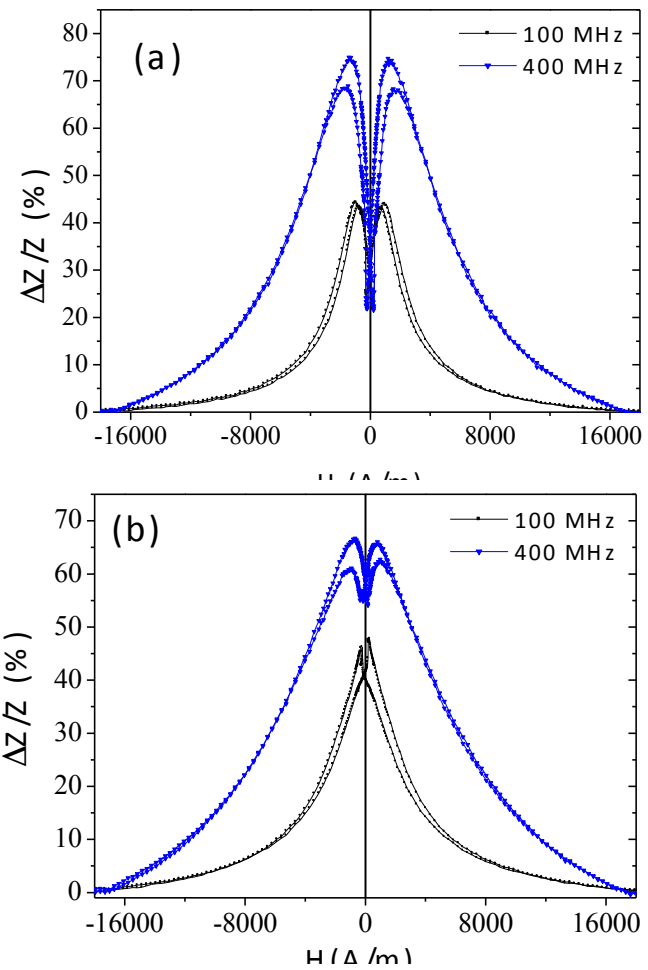

Figure 3: $\Delta \mathrm{Z} / \mathrm{Z}(\mathrm{H})$ dependences of as-prepared (a) and annealed at $200^{\circ} \mathrm{C}$ for $5 \mathrm{~min}$ (b) $\mathrm{Co}_{50.69} \mathrm{Fe}_{8.13} \mathrm{Ni}_{17.55} \mathrm{~B}_{13.29} \mathrm{Si}_{10.34}$ measured at different frequencies

The difference of $\Delta Z / Z(\mathrm{H})$ dependences measured for as-prepared (Fig.3a) and annealed (Fig.3b)samples is the value of the magnetic field, $H_{m}$, at which $\Delta Z / Z$ maximum takes place: for annealed samples $H_{m}$-values are lower than for as-prepared samples for all measured frequencies. Increasing the frequency, $f$, the maximum GMI ratio, $\Delta \mathrm{Z} / \mathrm{Z}_{\mathrm{m}}$, rises (see Fig.3).

Observed changes of the hysteresis loops after annealing must be associated with the stress relaxation. Indeed the magnetostriction constant depends on stresses (both applied and internal) in according the following equation:

$$
\lambda_{s}(\sigma)=\lambda_{s}(0)-B \sigma
$$

where $\lambda_{s}(\sigma)$ is the magnetostriction constant under stress; $\lambda_{s}(0)$ is the zero-stress magnetostriction constant; $B$ is a positive coefficient of order $10^{-10} \mathrm{MPa}$, and $\sigma$ stresses. Furthermore, as experimentally shown in Fig. 4, the magnetostriction considerably change after annealing to

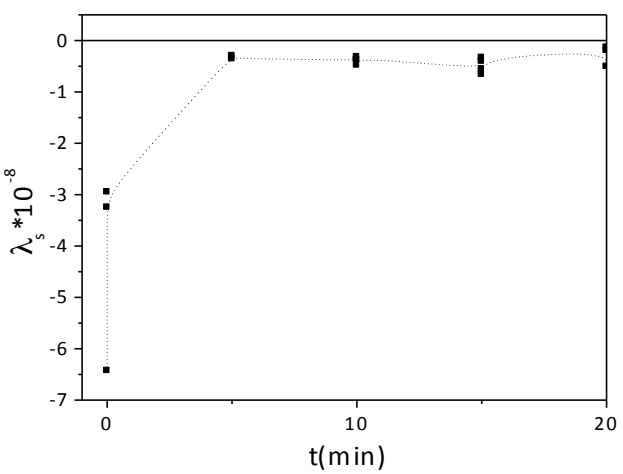

Figure 4: Dependence of the magnetostriction coefficient on annealing time for annealed $\mathrm{Co}_{69.2} \mathrm{Fe}_{4.1} \mathrm{~B}_{11.8} \mathrm{Si}_{13.8} \mathrm{C}_{1.1}$ microwire samples at $\mathrm{T}_{\mathrm{ann}}=$ $300^{\circ} \mathrm{C}$

nearly zero for annealed $\mathrm{Co}_{69.2} \mathrm{Fe}_{4.1} \mathrm{~B}_{11.8} \mathrm{Si}_{13.8} \mathrm{C}_{1.1}$ microwire samples after annealing at $t_{a n n} \geq 5 \mathrm{~min}$.

In addition, if the magnetostriction coefficient is low and negative, we must consider two opposite consequences of the internal stresses. The first contribution is an increase of the total magnetoelastic energy given by eq (3):

$$
K_{m e}=3 / 2 \lambda_{s} \sigma_{i},
$$

where $\lambda_{s}$ is the saturation magnetostriction and $\sigma_{\mathrm{i}}$ is the internal stress induced by the glass coating layer during the fabrication technique of glass-coated microwires.

The second one must be related to the stress dependence (either applied or internal stresses: $\sigma=\sigma_{\text {applied }}+\sigma_{i}$ ) on magnetostriction coefficient described by eq. 2, which is quite relevant for the case of low magnetostriction constant, $\lambda_{s, 0}$. Accordingly, the magnetostriction constant under stress, $\lambda_{s, \sigma}$, must decrease. Based on the dependencies observed in Figs.2-3, the increase of coercivity and rectangular character of hysteresis loops after annealing must be attributed to the stress relaxation and corresponding magnetostriction changes. We can also assume that the outer domain shell of the annealed Co-rich microwire that exhibits both rectangular hysteresis loop and a GMI effect has high circumferential magnetic permeability.

\subsection{Tailoring of GMI effect in nanocrystalline Fe-rich microwires}

Usually amorphous Fe-rich microwires present rectangular hysteresis loop as shown in Fig. 5 typical for Ferich amorphous microwires.

Typically Fe-rich amorphous microwires present relatively low GMI effect (1-2 order smaller than Co-rich microwire).

The conventional way of formation of the nanocrystalline structure from the amorphous state, is the heat treatment (with appropriate annealing conditions as temperature, annealing time, heating rate, etc.) $[14,15]$. The evolution of structural and magnetic properties of $\mathrm{Fe}_{70.8} \mathrm{Cu}_{1} \mathrm{Nb}_{3.1} \mathrm{Si}_{14.5} \mathrm{~B}_{10.6}$ microwire has been studied in at different annealing temperature in the range between $400-650^{\circ} \mathrm{C}$ for 1 hour in order to investigate the devitrification process (Fig. 6). 


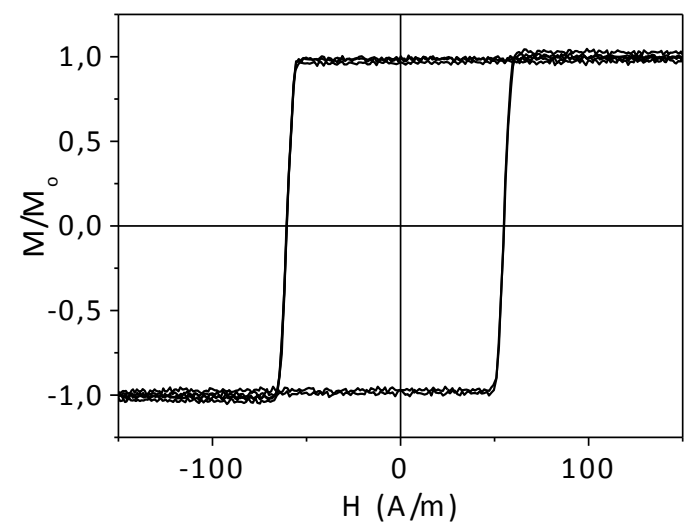

Figure 5: Hysteresis loops of as-prepared $\mathrm{Fe}_{70.8} \mathrm{Cu}_{1} \mathrm{Nb}_{3.1} \mathrm{Si}_{14.5} \mathrm{~B}_{10.6}$ microwires .

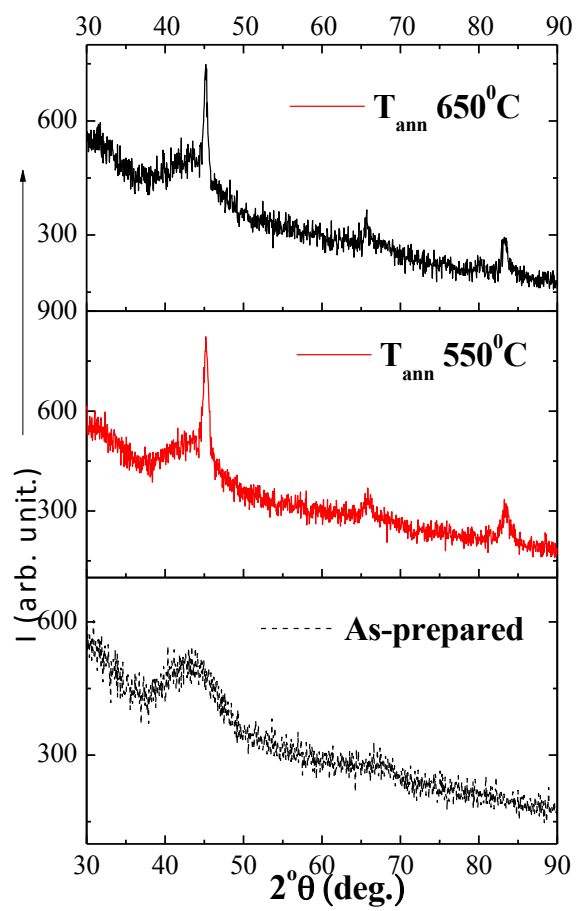

Figure 6: XRD patterns of as-prepared and annealed at different temperature for 1 hour $\mathrm{Fe}_{70.8} \mathrm{Cu}_{1} \mathrm{Nb}_{3.1} \mathrm{Si}_{14.5} \mathrm{~B}_{10.6} \quad$ glass-coated microwires.

Starting from $550^{\circ} \mathrm{C}$ to $650^{\circ} \mathrm{C}$ a main crystalline peak is appearing in the range between $42^{\circ}$ to $45^{\circ}$ which is correspond to the existence of $\alpha-\mathrm{Fe}$ (Si) BCC crystal structure $[13,14]$, as well as another two weak peaks appear in the range between $65^{\circ}$ to $85^{\circ}$.

The grain size has been estimated based on Scherrer equation as we previously reported [16]. From this analysis we can underline that the grain size of studied microwires increases from 17 to $22 \mathrm{~nm}$ upon increasing the annealing temperature from 550 to $650^{\circ} \mathrm{C}$, respectively.

The formation of fine nanograins within the amorphous matrix allows reducing the net magnetostriction, according to this equation:

$$
\lambda_{s, e f f}=V_{c r} \lambda_{s, c r}+\left(1-V_{c r}\right) \lambda_{s, a m}
$$

where $\lambda_{\mathrm{s}, \text { eff }}$ the saturation magnetostriction coefficient and $V_{c r}$ - the crystalline volume fraction.

Consequently, the enhanced soft magnetic properties of these kinds of material can be achieved. As can be observed from Fig.7 considerable magnetic softening is observed after samples annealing.

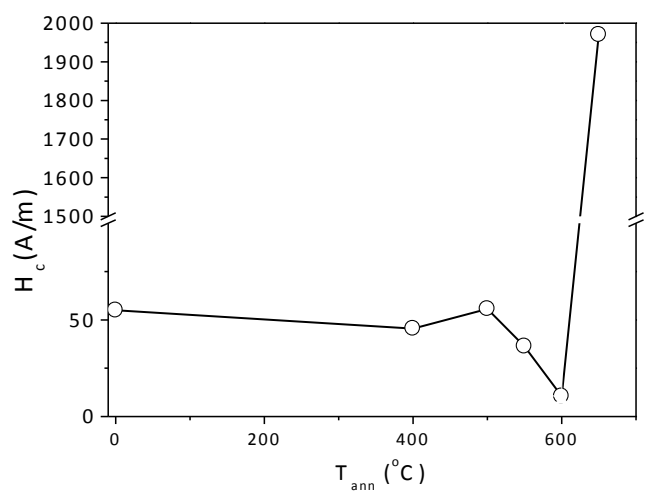

Figure 7: Coercivity dependence on annealing temperature measured in $\mathrm{Fe}_{70.8} \mathrm{Cu}_{1} \mathrm{Nb}_{3.1} \mathrm{Si}_{14.5} \mathrm{~B}_{10.6}$ microwire with $\rho=0.6$

Magnetic softening (optimum softest behavior) with quite low value of coercivity is obtained in the samples treated at $\mathrm{T}_{\text {ann }} \approx 500-600{ }^{\circ} \mathrm{C}$ which is ascribed to the fact that the first crystallization process has been developed, leading to fine nanocrystals $\alpha-\mathrm{Fe}(\mathrm{Si})$ of grain size around $10-20 \mathrm{~nm}$, such it has been widely reported for FINEMET- ribbons (see for example ref. 17). The precipitation of the second phase results in abrupt increase of the coercivity observed for annealing temperature above $600^{\circ} \mathrm{C}$.

Consequently together with magnetic magnetic softening we observed great enhancement of the GMI effect (see Fig.8) after devitrification of studied sample: maximum

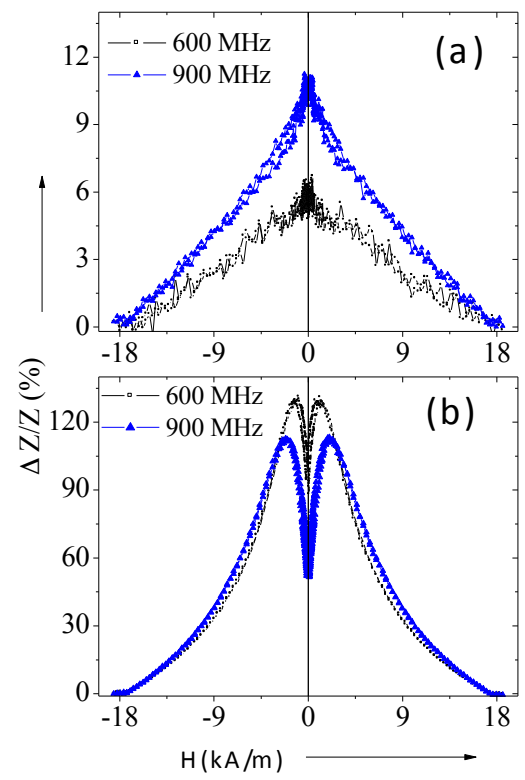

Figure 8: $\Delta \mathrm{Z} / \mathrm{Z}(\mathrm{H})$ dependences of as-prepared (a) and annealed at $\mathrm{T}_{\mathrm{ann}}=550^{\circ} \mathrm{C}(\mathrm{b})$ $\mathrm{Fe}_{70.8} \mathrm{Cu}_{1} \mathrm{Nb}_{3.1} \mathrm{Si}_{14.5} \mathrm{~B}_{10.6}$ microwires measured at different frequencies 
GMI ratio increases more than one order from $\approx 7 \%$ up to $130 \%$. Observed values of GMI effect in less expensive Ferich microwires with nanocrystalline structure are of practical interest for GMI related-applications.

It is worth mentioning that the main interest in amorphous soft magnetic materials is related to their liquidlike structure characterized by the absence of long range ordering and defects typical for crystalline magnetic materials. Therefore, the main parameter determining magnetic softness of amorphous materials is the magnetoelastic anisotropy. The role of the magnetostriction coefficient, $\lambda_{s}$, is already discussed above. But the other contribution is related to the internal stresses, $\sigma_{i}$. In the case of glass-coated microwires the main part of internal stress is induced by the glass coating during the simultaneous rapid solidification of metallic nucleus surrounded by glass coating during the glass-coated microwires preparation [6, $8,18]$. The strength of the internal stresses depends on the $\rho$ ratio between the metallic nucleus diameter, $\mathrm{d}$, and total microwire diameter, $D(\rho=d / D)[8,18]$ increasing with the the $\rho$-ratio decreasing. Therefore $\rho$-ratio is the other relevant parameter determining the magnetoelastic anisotropy.

On the other hand, magnetic softness of amorphous materials can be improved in the case of nanocrystallization at which a formation of two-phase systems consisting of nanocrystalline grains randomly distributed in a soft magnetic amorphous phase is observed [14-17]. After nanocrystallization, such material consists of small (around $10 \mathrm{~nm}$ grain size) nanocrystallites embedded in the residual amorphous matrix. Aforementioned magnetic softness is thought to be originated because the magnetocrystalline anisotropy vanishing and decreasing of the magnetostriction coefficient value which can achieve quite low magnetostriction value when the grain size approaches 10 nm [14-17].

Consequently we demonstrated that both annealing allowing internal stresses relaxation, modification of the magnetostriction coefficient by stress relaxation or by devitrification of the amorphous precursor can be used for engineering of magnetic properties and GMI effect of glasscoated micrtowires.

\section{Conclusions}

We showed that the GMI effect of magnetic wires can be tuned by annealing. We observed that annealing of amorphous Co-rich microwire considerably affects its hysteresis loop, GMI effect. The observed dependences of these characteristics are attributed to stress relaxation and changes in the magnetostriction after sample annealing.

Considerable magnetic softening and increasing of the GMI effect is observed in less expensive Fe-rich nanocrystalline FINEMET-type glass-coated microwires after the devitrification process caused by thermal annealing.

\section{Acknowledgements}

This work was supported by Spanish MINECO under MAT2013-47231-C2-1-P and the Russian Science Foundation under the 16-19-10490 grant. Technical and human support provided by SGIker (UPV/EHU, MICINN, GV/EJ, ERDF and ESF) is gratefully acknowledged. A. Zhukov and V. Zhukova wish to acknowledge support of the Basque Government under Program of Mobility of the Investigating Personnel of the Department of Education, Universities and Investigation (Grants MV_2017_1_25 and MV_2017_1_30 respectively).

\section{References}

[1] D.P. Makhnovskiy, L.V. Panina, C. Garcia, A.P. Zhukov, and J. Gonzalez, Experimental demonstration of tunable scattering spectra at microwave frequencies in composite media containing $\mathrm{CoFeCrSiB}$ glass-coated amorphous ferromagnetic wires and comparison with theory, Phys. Rev. B, Vol. 74 No. 6: 2006.

[2] M. Ipatov, V. Zhukova, A. Zhukov and L. V. Panina, Microwave Metamaterials Containing Magnetically Soft Microwires, Advances in Science and Technology, 75: 224-229, 2010.

[3] F.X. Qin, H.X. Peng, M.H. Phan, L.V. Panina, M. Ipatov, A. Zhukov, Effects of wire properties on the fieldtunable behaviour of continuous-microwire composites, Sensors and Actuators A 178: 118- 125, 2012.

[4] L.V. Panina and K. Mohri, Magneto-impedance effect in amorphous wires," Appl. Phys. Lett., 65: 1189-1191, 1994.

[5] A. Zhukov, A. Talaat, M. Ipatov and V. Zhukova, Tailoring the high-frequency giant magnetoimpedance effect of amorphous Co-rich microwires, IEEE Magn. Lett., 6: 2500104, 2015.

[6] A. Zhukov, M. Ipatov and V. Zhukova, Advances in Giant Magnetoimpedance of Materials, Handbook of Magnetic Materials, ed. K.H.J. Buschow, 24: chapter 2, 139-236, 2015

[7] D.C. Jiles, Recent advances and future directions in magnetic materials. Acta Mater. 51: 5907-5939, 2003

[8] H. Chiriac, T. A. Ovari, and Gh. Pop, Internal stress distribution in glass-covered amorphous magnetic wires, Phys. Rev. B 42: 10105-10113, 1995.

[9]J. M. Blanco, A. Zhukov and J. Gonzalez, Effect of tensile and torsion on GMI effect in amorphous wire, $J$. Magn. Magn. Mat. 196-197: 377-379, 1999.

[10]J.M. Blanco, A. Zhukov and J.Gonzalez, Torsional Stress Impedance and Magneto-impedance in $\left(\mathrm{Co}_{0.95} \mathrm{Fe}_{0.05}\right)_{72.5} \mathrm{Si}_{12.5} \mathrm{~B}_{15}$ Amorphous Wire with Helical Induced Anisotropy, J. Phys. D:Appl. Phys. 32: 31403145, 1999.

[11] K. Narita, J. Yamasaki and H. Fukunaga, IEEE Trans.Magn. , Measurement of Saturation Magnetostriction of a Thin Amorphous ribbon by Means of Small-Angle Magnetization Rotation, 16: 435-439, 1980.

[12] A. Zhukov, M. Churyukanova, S. Kaloshkin, V. Sudarchikova, S. Gudoshnikov,M. Ipatov, A. Talaat, 
J.M. Blanco and V. Zhukova, Magnetostriction of CoFe-based amorphous soft magnetic microwires, $J$. Electr. Mater. 45: Issue 1, 226-234, 2016

[13] A. Zhukov, J. M. Blanco, M. Ipatov, A. Chizhik and V. Zhukova, Manipulation of domain wall dynamics in amorphous microwires through the magnetoelastic anisotropy, Nanoscale Research Letters, 7: 223, 2012.

[14] Y. Yoshizawa and K. Yamauchi, Fe-based soft magnetic alloy composed of ultrafinegrain structure, Materials transaction JIM, 31: 307-314, 1990.

[15] A.Zhukov, K.Chichay, A.Talaat, V.Rodionova, J.M.Blanco, M.Ipatov, V. Zhukova, Manipulation of magnetic properties of glass-coated microwires by annealing, J. Magn. Magn. Mater. 383: 232-236, 2015

[16] A. Zhukov, A. Talaat, M. Ipatov, J. J. del Val, L. Gonzalez-Legarreta, B. Hernando and V. Zhukova, Effect of nanocrystallization on magnetic properties and GMI effect of Fe-rich microwires, J. Electr. Mater. 43, No. 12: 4540-4547, 2014

[17] ] H. Q. Guo, H. Kronmüller, T. Dragon, Z. H. Cheng, and B. G. Shen, Influence of nanocrystallization on the evolution of domain patterns and the magnetoimpedance effect in amorphous Fe73.5Cu1Nb3Si13.5B9 ribbons, J. Appl. Phys., 89: 514-520, 2001.

[18] A. Zhukov, J. Gonzalez, A. Torcunov , E. Pina, M.J Prieto, A. F. Cobeño, J.M. Blanco, V. Larin and S. Baranov, Ferromagnetic resonance and Structure of Febased Glass-coated Microwires, J. Magn. Magn. Mater. 203: 238-240, 1999. 